\title{
Spin-resolved two-photon photoemission study of the surface resonance state on $\mathrm{Co} / \mathrm{Cu}(001)$
}

\author{
O. Andreyev, ${ }^{1}$ Yu. M. Koroteev, ${ }^{2,3}$ M. Sánchez Albaneda, ${ }^{1}$ M. Cinchetti, ${ }^{1}$ G. Bihlmayer, ${ }^{4}$ E. V. Chulkov, ${ }^{2,5}$ J. Lange, ${ }^{1}$ \\ F. Steeb, ${ }^{1}$ M. Bauer, ${ }^{1, *}$ P. M. Echenique, ${ }^{2,5}$ S. Blügel, ${ }^{4}$ and M. Aeschlimann ${ }^{1}$ \\ ${ }^{1}$ Department of Physics, University of Kaiserslautern, Erwin Schroedinger-Str. 46, 67663 Kaiserslautern, Germany \\ ${ }^{2}$ Donostia International Physics Center (DIPC), 20018 San Sebastián, Spain \\ ${ }^{3}$ Institute of Strength Physics and Materials Science, Russian Academy of Sciences, 634021, Tomsk, Russia \\ ${ }^{4}$ Institut für Festkörperforschung, Forschungszentrum Jülich, 52425 Jülich, Germany \\ ${ }^{5}$ Departamento de Física de Materiales and Centro Mixto CSIC-UPV/EHU, Facultad de Ciencias Químicas, UPV/EHU Apdo 1072, \\ 20080 San Sebastián/Donostia, Spain \\ (Received 16 February 2006; revised manuscript received 1 October 2006; published 14 November 2006)
}

\begin{abstract}
Bulk and surface states of a clean and Cs-doped surface of a Co film grown on $\mathrm{Cu}(001)$ have been studied by spin-resolved photoemission (SR-PE) and compared with band structure calculation results. One-photon (1PPE) and two-photon (2PPE) photoemission spectra from clean Co films are found to be dominated by a peak located at a binding energy of about $0.4 \mathrm{eV}$ with respect to $\mathrm{E}_{\mathrm{F}}$, which is assigned to the spin up $3 d$ bulk state. Slight Cs-doping of a Co(001) surface shifts an image potential state in resonance with the $s p$-states of the conduction band. SR-2PPE study of the optically-induced electron population in such an image resonance reveals a strong dependence on the set polarization of the laser light. We are able to directly detect the spin polarization of electrons photoemitted from the image resonance state, which can be varied from highlypolarized (about bulk values) to almost unpolarized when tuning light polarization of the pump laser pulse from $s$ to $p$.
\end{abstract}

DOI: 10.1103/PhysRevB.74.195416

PACS number(s): 79.60.-i, 73.20.At

\section{INTRODUCTION}

Spintronics is a fast-developing field, which is based on the manipulation of the spin degree of freedom. ${ }^{1,2}$ Successful application of spin-based devices requires a thorough clarification of the spin polarization transport and injection in solids. Ferromagnets are commonly used as an intrinsic source of the spin-polarized charge carriers. The principle of operation of devices such as spin valves or magnetic tunnel junctions is based on the electronic band structure, which is dependent on the spin direction. ${ }^{1,2}$ Naturally, an understanding of their occupied and unoccupied magnetic bulk and surface/ interface states is essential.

Surface states attract considerable interest as they extend the fundamental knowledge about surface electronic structure. Their wave functions are strongly coupled to the surface band structure of the crystal, and they can serve as a convenient probe for studying electronic structure and dynamics at surfaces. Several authors presented the possibility to investigate surface ferromagnetism and magnetization dynamics by probing the state of crystal-induced or image-potential surface states. ${ }^{3-6}$

In a two-photon photoemission (2PPE) study, Wallauer and Fauster (Ref. 4) showed that the spin-splitting of the ferromagnetic image state can be effectively used to demonstrate that the top surface layer of a $\mathrm{Fe}(001)$ surface is ferromagnetic, an issue that had been under discussion for many years. The sophisticated use of dipole selection rules determining the spin-dependent transition probabilities between the bulk valence band and the image state play a key role in this study.

In this paper, we show that these properties make it possible to control the degree of spin polarization of an optically excited surface state population. We observe that by rotating the electric field vector of the exciting light, the spin polarization degree of a $\mathrm{Co}(001)$ surface resonance can be tuned from unpolarized to more than $40 \%$, the value of the Co bulk spin polarization. Parallel to our experimental findings, $a b$ initio calculation of the $\mathrm{Co}(001)$ and surface band structure are exploited.

This paper starts with a brief introduction into the spinresolved 2PPE technique which has been used in this work to probe the spin polarization of the $\mathrm{Co}(001)$ excited surface resonance. Details of the occupied electronic band structure of $\mathrm{Co}(001)$ as revealed by spin-resolved 2PPE under consideration of band structure calculations are discussed in comparison to conventional PE results in the following section. These electronic bulk states act as initial states for the photoinduced population of a $\mathrm{Co}(001)$ surface resonance which becomes accessible in our experiment after a moderate lowering of the work function by adsorption of cesium. Our spin-resolved data using $p$ - and $s$-polarized light is discussed in the final section of this paper.

\section{EXPERIMENT}

The experimental set-up for our studies is similar to the one reported in Knorren et al. (Ref. 7). In brief, a femtosecond Ti:Sapphire laser is used as a pulsed light source. The system delivers $\mathrm{sech}^{2}$ temporal shaped pulses of up to $10 \mathrm{~nJ} /$ pulse at a repetition rate of about $80 \mathrm{MHz}$ covering a wavelength regime between $730 \mathrm{~nm}$ and $890 \mathrm{~nm}$. The linearly polarized laser output is frequency doubled in a $0.2 \mathrm{~mm}$ thick $\beta$-barium-borate crystal (BBO) to produce light pulses of $\mathrm{h} \nu \approx 3.1 \mathrm{eV}$. Both fundamental (IR) and second harmonic (UV) laser light can be focused onto the sample which is mounted in an ultrahigh vacuum (UHV) chamber with a base pressure below $10^{-10}$ mbar. The high 
peak intensity of the laser pulses enables the 2PPE of electrons from the surface. This technique is sensitive to both occupied as well as unoccupied states of the surface. In consideration of the dipole selection rules, this method also allows insight into the coupling between these states. As a typical photoemission method, 2PPE is intrinsically sensitive to surface states such as image-potential states, Shockley states or Tamm states. ${ }^{8}$ On the other hand, due to the enlarged mean free path of the low energetic electrons involved in the 2PPE process, this method has also been applied successfully in the investigation of bulk electronic properties. ${ }^{9,10}$

The photoemitted electrons are analyzed by a commercial cylindrical sector analyzer (Focus CSA 300) equipped with an additional detector for spin-polarized electrons based on spin-polarized low-energy electron diffraction (FocusSPLEED). The energy resolution was set to roughly $50 \mathrm{meV}$ resolution (closed entrance slit) for the spin-integrated measurements, and $100 \mathrm{meV}$ to $150 \mathrm{meV}$ (open entrance slit) for the spin-resolved measurements. The acceptance angle of the analyzer is $\pm 13^{\circ}$. The orientation of the sample is $45^{\circ}$ with respect to the laser beam, and electrons are detected in the normal-emission geometry.

Several degrees of freedom provided by our optical set-up of the experiment allow a high flexibility in performing the 2PPE experiments. So-called monochromatic (UV-UV) and bichromatic (IR-UV) experiments can be performed in which two separate light pulses are incident onto the surface, thus inducing the photoemission. The orientation of the polarization vector of both beams can be adjusted independently by the use of zero-order waveplates. Finally, to be able to perform one-photon photoemission (1PPE) experiments, we use the fourth harmonic of the laser output $(\mathrm{h} \nu=6.1 \mathrm{eV})$ generated by sequential frequency doubling in two BBO crystals.

The Co(001) surface was prepared by epitaxial growth of a Co film onto a $\mathrm{Cu}(001)$ substrate. Prior to evaporation the $\mathrm{Cu}(001)$ crystal was routinely cleaned by repeated argon ion sputtering and annealing $(500 \mathrm{~V}, 750 \mathrm{~K})$. Evaporation of $2 \mathrm{~nm}$ to $10 \mathrm{~nm}$ thick Co films is performed in situ in a separate chamber at a base pressure of $6 \times 10^{-11} \mathrm{mbar}$. The film thickness was measured with a quartz oscillator thickness monitor which has been calibrated against atomic-forcemicroscopy measurements. Co films were grown quasiepitaxially on the $\mathrm{Cu}(001)$ surface in a metastable tetragonally-distorted fcc [face centered tetragonal (fct)] structure with in-plane uniaxial magnetic anisotropy. ${ }^{11}$ After evaporation, the Co film was annealed at $440 \mathrm{~K}$ to produce a defect-free smooth surface. Cu contamination of the Co surface is ruled out for the prepared samples. Diffusion of $\mathrm{Cu}$ atoms through pinholes is a well-known process found in ultrathin Co films (few ML thick) annealed at temperatures $>450 \mathrm{~K} \cdot{ }^{12}$ At a film thickness of up to $55 \mathrm{ML}$ and a maximum annealing temperature of $440 \mathrm{~K}$ this process is not relevant for the present work. In addition, we have never observed any indication of $\mathrm{Cu}$ in the Auger spectra. Prior to the photoemission measurements the films were magnetized in an external field oriented along the in-plane easy direction. Cs doping is done from a well-degassed Cs-getter source (SAES). All experiments were performed at room temperature.

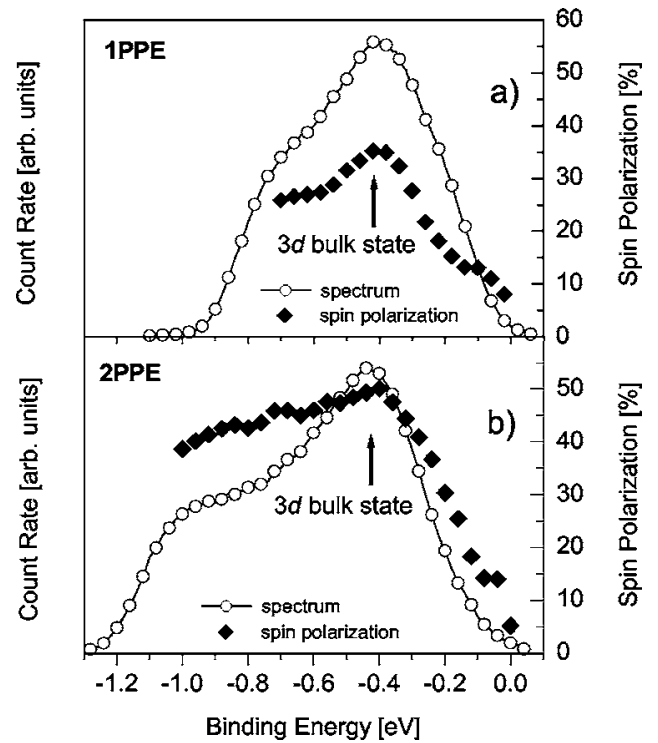

FIG. 1. (a) $1 \mathrm{PPE}(\mathrm{h} \nu=6.1 \mathrm{eV})$ and (b) $2 \mathrm{PPE}(\mathrm{h} \nu=3.2 \mathrm{eV})$ spectra and corresponding spin polarization of a $\mathrm{Co}(001)$ surface obtained with $p$-polarized light.

\section{THEORETICAL METHOD}

The calculations were performed using density functional theory in the local density approximation (LDA) in the form of Moruzzi et al. ${ }^{13}$ We use the full-potential linearized augmented plane-wave method $^{14,15}$ as implemented in the FLEUR code. ${ }^{16}$ For the calculations a cutoff of $\mathrm{R}_{\mathrm{MT}} \times \mathrm{K}_{\max }$ $=2.25 \times 3.8$ was used and the two-dimensional Brillouinzone was sampled with 28 special $k$-points. We calculated a 13 layer fct $\mathrm{Co}(001)$ film with an in-plane lattice constant of $2.56 \AA$ as would be found on a $\mathrm{Cu}(001)$ substrate. The interlayer distance, $d=1.70 \AA$, was deduced from a total energy minimization provided that the in-plane fct Co lattice constant coincides with the fcc $\mathrm{Cu}(001)$ lattice parameter. The position of the top three layers was allowed to relax in the direction of the surface normal. As compared to the ideal interlayer distance, the first interlayer spacing contracted by $4.8 \%$, while the distance between the second and the third layer expanded by $2.6 \%$. The contraction of the third interlayer spacing was small $(0.36 \%)$.

\section{RESULTS AND DISCUSSION}

\section{A. Spin up 3d bulk state}

1PPE and monochromatic 2PPE spectra using $p$-polarized light and the measured spin polarizations of the pure Co film are presented in Fig. 1. The electrons photoemitted from the surface are spin-polarized as result of the spin-dependent electron density of states of the itinerant ferromagnet cobalt. Both 1PPE and 2PPE spectra are dominated by a peak located at a binding energy of about $0.4 \mathrm{eV}$ with respect to $E_{F}$. For the whole energy range, a prevailing majority-spin polarization is found exhibiting a maximum that coincides with the peak in the photoemission spectra. In 1PPE, we find 


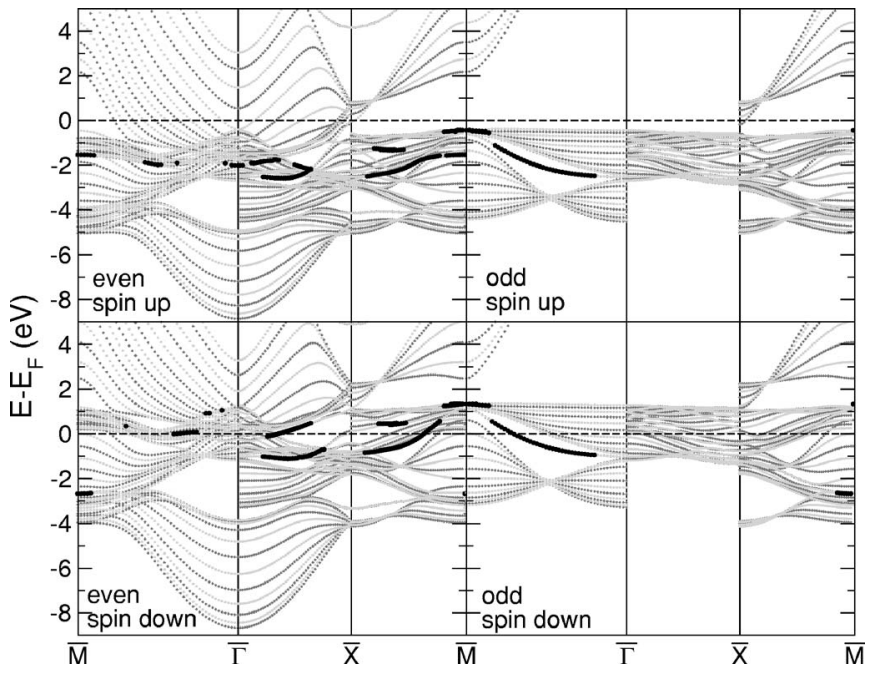

FIG. 2. Surface electronic structure of the fct $\mathrm{Co}(001)$ surface as obtained from the calculation of a relaxed 13 layer fct $\mathrm{Co}(001)$ film with a $\mathrm{Cu}(001)$ in-plane constant. The left and right panels show states with even and odd symmetry with respect to a mirror plane that is parallel to the $k$-vector and perpendicular to the surface. Dark and light gray symbols indicate the symmetry with respect to z-reflection. Surface states, i.e., states that have more than $50 \%$ weight in the surface layer, are marked by black circles.

maximum values in the spin polarization of $35 \%$. In case of the $2 \mathrm{PPE}$ this value exceeds even $45 \%$. This tendency of an increased spin polarization in case of $2 \mathrm{PPE}$ in comparison to $1 \mathrm{PPE}$ is well understood and can be assigned to an enhanced spin-filter effect in the 2PPE process, where electrons experience a spin-dependent electron scattering not only in the final but also in the intermediate state. ${ }^{17}$ Note that close to $E_{F}$ in both cases a clear drop in the photoemission yield and in the spin polarization down to a value of about $10 \%$ is observed.

For comparison with these experimental data, we display in Figs. 2 and 3 the calculated surface band structure and local (layer) density of states of $\mathrm{Co}(001)$ for the majority and minority electrons.

The upper and lower panel of Fig. 3 shows the result for a surface and central (bulk) layer, respectively, after the integration over a small fraction (1/9th) of the Brillouin zone around the $\bar{\Gamma}$ point in order to mimic the finite angular resolution of our electron energy analyzer. For normal emission, the detected electrons cover a momentum spread corresponding to an upper limit of about $1 / 5$ th of the BZ. The LDOSdata integrated over 1/9th of the BZ show indeed a high peak in the spin up DOS for the central layer that coincides in binding energy almost perfectly with the peak position found in the 1PPE and 2PPE spectra. The calculations give a value of $0.45 \mathrm{eV}$ in comparison to $0.4 \mathrm{eV}$ determined in the experiment. Note that the peak for the central layer in the calculated DOS becomes much less pronounced when performing the $k$ integration over a much wider area of the BZ. A corresponding feature is not present in case of an integrated spindown DOS which agrees with the experimental observation

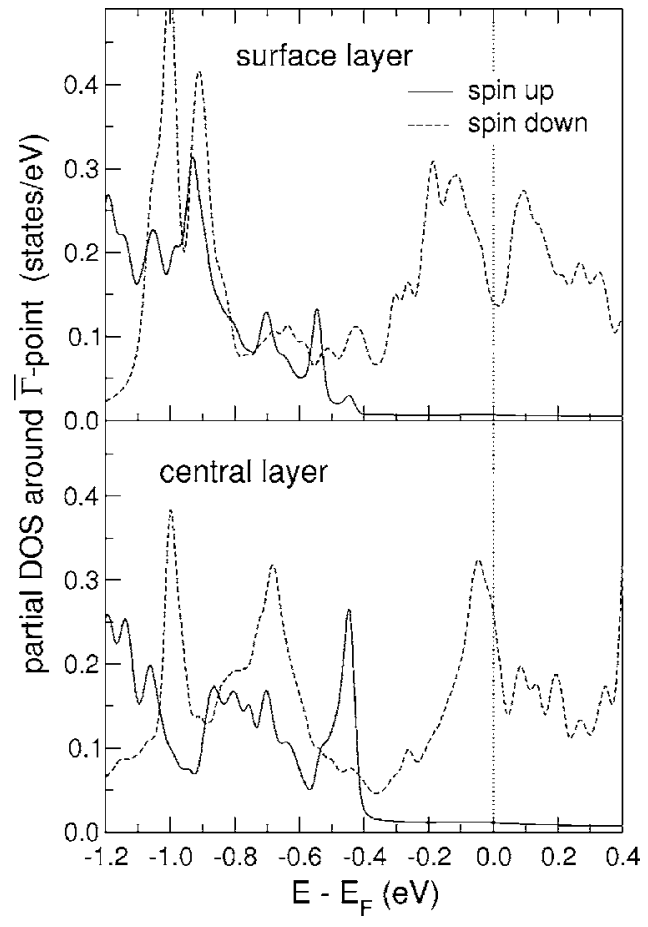

FIG. 3. Density of states for the surface (top) and central (bottom panel) layer of a 13 layer fct $\mathrm{Co}(001)$ film. The calculation of the layer density of states was performed by integrating over $1 / 9$ of the Brillouin zone in an area around the $\bar{\Gamma}$ point.

of an increase in the majority spin polarization right at the energy of the spectral peak.

In the spin-resolved photoemission study of $\mathrm{Co}(001)$ using an ultraviolet synchrotron radiation $(\mathrm{h} \nu=45 \mathrm{eV})$, Clemens et al. ${ }^{18,19}$ observed a spin up peak at a binding energy of $0.6 \mathrm{eV}$ that was assigned to the emission from a $3 d$ surface state or resonance. The calculated DOS of a surface layer of $\mathrm{Co}(001)$ as shown in the upper part of Fig. 3 does not support such an interpretation for the present case. In the relevant energy region $(0.4 \mathrm{eV}$ binding energy) no surface lo-

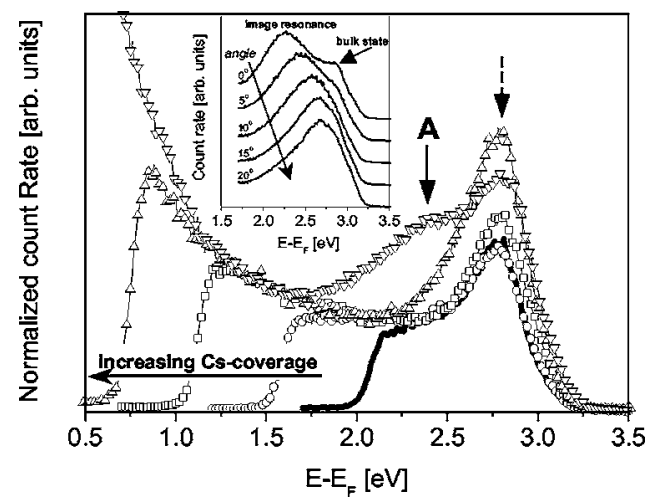

FIG. 4. Monochromatic 2PPE spectra of a clean Co(001) surface (solid line) and $\mathrm{Co}(001)$ surface at varying cesium coverage (symbols) obtained with $p$-polarized light $(\mathrm{h} \nu=3.2 \mathrm{eV})$. The dashed arrow shows the energy position of the bulk state that is not moving due to the adsorption of Cs. Inset: Angle-dependence of the image resonance state. 
calized state can be identified. Furthermore, we observe in our experiment that the energy and intensity of the peak are rather insensitive to adsorption of cesium (see dashed arrow in Fig. 4). Due to the high polarizability of alkali atoms at a surface, it is well known that the adsorption of cesium should result in a clear change of the surface potential and, consequently, the surface electronic structure. We therefore conclude that the dominant peak observed in $1 \mathrm{PPE}$ and $2 \mathrm{PPE}$ close to $E_{F}$ is due to photoemission from the spin up $3 d$ bulk band $\left(\Delta_{5}\right.$ bulk peak). However, photoemission is an intrinsically surface sensitive technique. The DOS of a surface layer must then have a significant impact on the photoemission yield. This may explain the large broadening of the $\Delta_{5}$ bulk peak in comparison with the peak in the calculated DOS for the central layer. In addition, the width of the calculated peak depends slightly on the integration interval over the $\bar{\Gamma}$ point. An integration over a wider area of the BZ results in a further broadening of the peak. Next to this peak, our calculations can qualitatively also reproduce the decrease in the photoemission yield and the spin polarization $P$ toward $E_{F}$ which is caused by the reduction of the $3 d$ majority-spin density of states in the strong ferromagnet Co due to the presence of the Stoner gap. An important requirement for a correct interpretation is, however, that the finite angular resolution of the experiment is considered in the calculated DOS.

We have also performed further investigations of the Co film, such as a tuning of the photon energy and angleresolved 2PPE measurements. ${ }^{20}$ These studies prove that the Co 2PPE spectrum is dominated by the revealed bulk state peak. We have not observed any additional contribution from unoccupied states in the studied energy region.

\section{B. Spin-selective excitation of the surface resonance}

In order to access lower lying intermediate states in these low-energy photoemission experiments we decreased the work function of the Co surface by slight alkali-doping (dosage $<0.2 \mathrm{ML}){ }^{21}$

Figure 4 shows 2PPE spectra recorded with $p$-polarized light from the clean Co surface and the Cs-covered Co surface at different Cs coverages up to a maximum of about 0.2 ML. The spectra are plotted as a function of the intermediate state energy. The downshift in the low energy onset of the 2PPE spectrum is due to the successive decrease in the work function induced by the Cs adatoms. For the maximum Cs coverage the work function has been lowered to a value of $3.5 \mathrm{eV}$ extending the accessible spectral regime by about $1.7 \mathrm{eV}$ in comparison to the clean Co surface. In this energy regime the 2PPE spectra show a pronounced rise in the photoemission yield towards small intermediate state energies. This rise is attributed to the high lifetime of electronic excitations at these energies which enhance the probability for $2 \mathrm{PPE}$ in comparison to the high energetic states. ${ }^{7}$ Additionally, the refilling of these states by secondary electrons (cascade electrons and Auger electrons) results in a stronger impact on the 2PPE yield the closer the intermediate state is located to $E_{F}{ }^{22}$

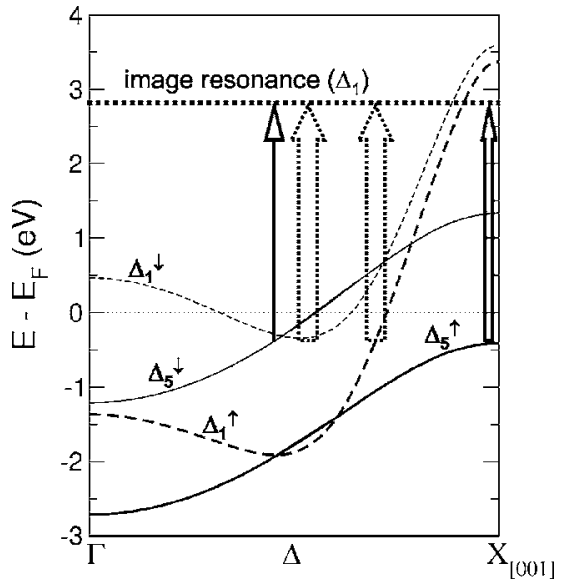

FIG. 5. Band structure of the fct Co along the $\Gamma \mathrm{X}_{[001]}$ direction. The arrows show allowed excitations from $\Delta_{5}$ (solid arrows) and $\Delta_{1}$ (dashed arrows) bands, according to Wallauer and Fauster, and Thomann et al. (Refs. 4 and 27).

Another spectral modification due to the alkali adsorption is noticeable at the high energy part of the spectra. The 2PPE intensity at the $\Delta_{5}$ bulk peak becomes seemingly larger and finally, for the highest Cs coverages, a new peak (A) appears just below the $\Delta_{5}$ bulk peak.

In order to study the origin of these spectral changes we examined the dependence of the energy of peak A on the variable photon energy $\mathrm{h} \nu$ of the used laser light. ${ }^{20}$ In the kinetic energy distribution we observe an energy shift of the peak indicative for an intermediate (excited) state. ${ }^{23}$ Angleresolved spectra shown in the inset of Fig. 4 demonstrate furthermore a significant energy dispersion of the peak as a function of the detection angle $\left(k_{\|}\right)$. For a large number of different alkali adsorption systems an unoccupied state has been identified in this energy regime which is in general assigned to an atomic orbital of the adsorbed alkali species. However, for low coverages, repulsive dipole-dipole interaction between neighboring alkali atoms hinders wave-function overlap so that this state is rather localized and therefore characterized by a missing energy dispersion. ${ }^{24}$ On the other hand, image potential states are delocalized states in the surface plane and therefore exhibit a significant upwards dispersion in energy (see inset of Fig. 4). These states are linked to the vacuum energy and can be found at binding energies of several hundred meV. For typical workfunctions between $4 \mathrm{eV}$ and $5.5 \mathrm{eV}$ in case of a clean surface, these states cannot be addressed in 2PPE using laser light at $\mathrm{h} \nu=3.1 \mathrm{eV}$. However, the reduction of the workfunction by the alkali dosage results in a corresponding downward shift of the image state energy so that at some point they also become accessible with the photon energies used for the present study.

We therefore assign this peak to the $n=1$ image state of the $\mathrm{Co}(001)$ surface. However, due to the downward shift, we expect the nature of this state to be significantly modified. The energy of the image state determined from the spectra in Fig. 4 is in resonance with the $s p$ states of the conduction band of the $\mathrm{Co}(001)$ surface (see band structure in Fig. 5), whereas for the pure $\operatorname{Co}(001)$ surface it is located within a 


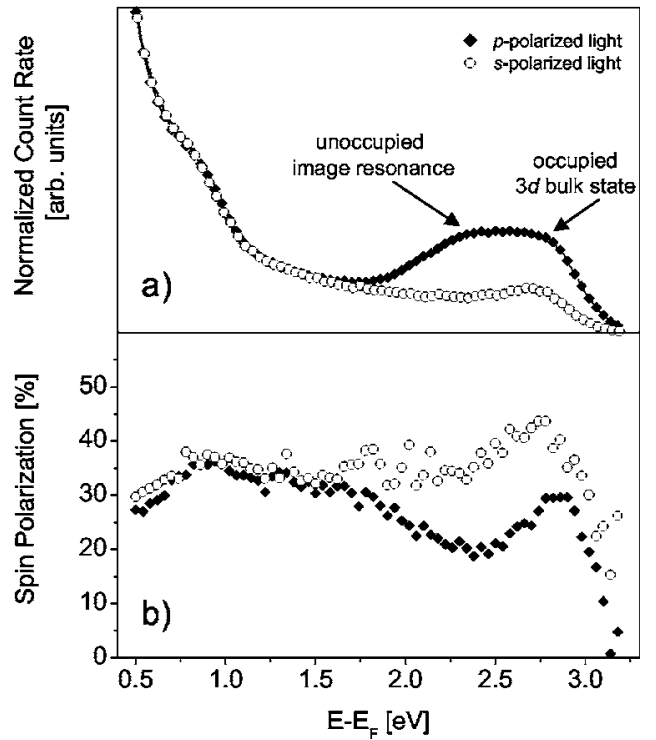

FIG. 6. Monochromatic 2PPE a) spectra and corresponding b) spin polarization of a Cs-covered $\operatorname{Co}(001)$ surface obtained with $p$ and $s$-polarized light $(\mathrm{h} \nu=3.2 \mathrm{eV})$.

bulk band gap. The coupling to the bulk band results in an efficient peak broadening of the image state, which is likely to be further enhanced by the interaction with the adsorbed cesium. Consequently, it has to be regarded as an image state resonance rather than an image state. ${ }^{23}$

In Fig. 6, 2PPE spectra from a Cs-doped Co surface and the corresponding spin polarization of the photoemitted electrons recorded with $s$ - and $p$-polarized light are compared. The two spectra differ mainly in the missing image state resonance for excitation with the $s$-polarized light. This selectivity on light polarization in the 2PPE process can be traced back to dipole selection rules for the involved transitions. In a normal emission, the final state of the PE process has to be totally symmetric with respect to all symmetry operations along the surface normal. ${ }^{25,26}$ In accord with $\Delta_{1}$ symmetry of the image potential state located inside of the band gap, we identify the image resonance with $\Delta_{1}$ symmetry. ${ }^{4}$ Therefore, a probing of the image resonance is only possible using $p$-polarized light. In this case, a component of the electric field vector normal to the surface is available which does not occur in the case of $s$-polarized light. Correspondingly, the 2PPE with $s$-polarized light involves exclusively bulk states, but no surface state. The variation of the light polarization from $s$ to $p$ therefore enables us to separate the surface contribution from the bulk photoemission spectrum of $\mathrm{Co}(001)$ as shown in Fig. 6(a).

The involvement of the image resonance in the 2PPE process performed with $p$-polarized light also affects the measured spin polarization. In comparison to an excitation with $s$-polarized light, a polarization drop of about $10 \%$ is found for $p$-polarized light in coincidence with the energy of the image resonance. This reduction in spin polarization is a consequence of the selection rules for the first optical transitions, i.e., the initial pump step of the 2PPE governing the population of the image resonance must be responsible for the decrease of spin polarization. Based on considerations by Wallauer and Fauster (Ref. 4) regarding a similar system, we discuss the relevant mechanisms to clarify our observation. As shown in Fig. 5 for $p$-polarized light, dipole selection rules enable only bands of $\Delta_{1}\left(s p\right.$-like) and $\Delta_{5}(d$-like) symmetry to participate in the excitation of the surface resonance state $\left(\Delta_{1}\right.$ symmetry) ${ }^{4,25,26}$ An optical excitation from occupied states of the same ( $s p$-like) character is preferential. Hence, a population of the surface resonance from the states with $\Delta_{1}$ symmetry is favoured in comparison to the excitation from $d$-like bands of $\Delta_{5}$ symmetry.

In addition, an optical coupling between initial and intermediate states also depends on the wave vector of the bands, which participate in excitations. Due to the same periodicity of the wave functions of the surface resonance and bulk bands at the $X$ point, there is a larger probability of excitation from the bands at high wave vectors than close to the $\Gamma$ point. ${ }^{4,27}$ However, in the relevant excitation region the wave vector and wave function of the $\Delta_{1}$ band are very similar for both spin up and spin down direction (excitations from these $\Delta_{1}$ bands are indicated by the dashed arrows in Fig. 5). We conclude that also the matrix elements for the optical transitions from spin up and spin down states to the surface resonance are very similar. Hence, nearly the same excitation intensities from spin up and spin down $\Delta_{1}$ bands will result in a low spin polarization in the electron population in the surface resonance.

Considering unfavoured excitation from $d$-like bands of $\Delta_{5}$ symmetry as compared to $s p$-like bands of $\Delta_{1}$ symmetry when using $p$-polarized light (see above), the spin polarization of the surface resonance must be small. We even find that the image resonance-intrinsic spin polarization approaches a value close to zero. The residual spin polarization measured at this energy is due to the bulk electron contribution to the 2PPE spectrum, which is of the same order as the image resonance contribution (see $s$-polarized 2PPE spectrum and spin polarization in Fig. 6).

A controlled variation of the Cs-doping level of the Co surface enabled us to study the image resonance at different intermediate state energies between $2.3 \mathrm{eV}$ and $2.8 \mathrm{eV}$ above $E_{F}{ }^{20}{ }^{20}$ In the entire energy range probed we found a similar intensity contribution of the image resonance to the $2 \mathrm{PPE}$ yield. Independent of the energy, we observed the (preferentially) same unpolarized pumping of the surface resonance with $p$-polarized light. Investigations at lower intermediate state energies of the image resonance (higher Cs coverage) were not possible, as the strong contribution of $1 \mathrm{PPE}$ at the corresponding low work function completely distorted the 2PPE part of the spectrum.

Let us now approach the case of the image resonance population induced by $s$-polarized light. The lack of a component of the electric field vector normal to the surface in this case enables excitation of the image resonance from the $3 d$ bands of $\Delta_{5}$ symmetry only. ${ }^{25,26}$ Those $3 d$ bands are responsible for the ferromagnetism in Co, and their DOS strongly depends on the electron spin direction. $d$ bands can yield high spin polarization of the photoemitted electrons up 


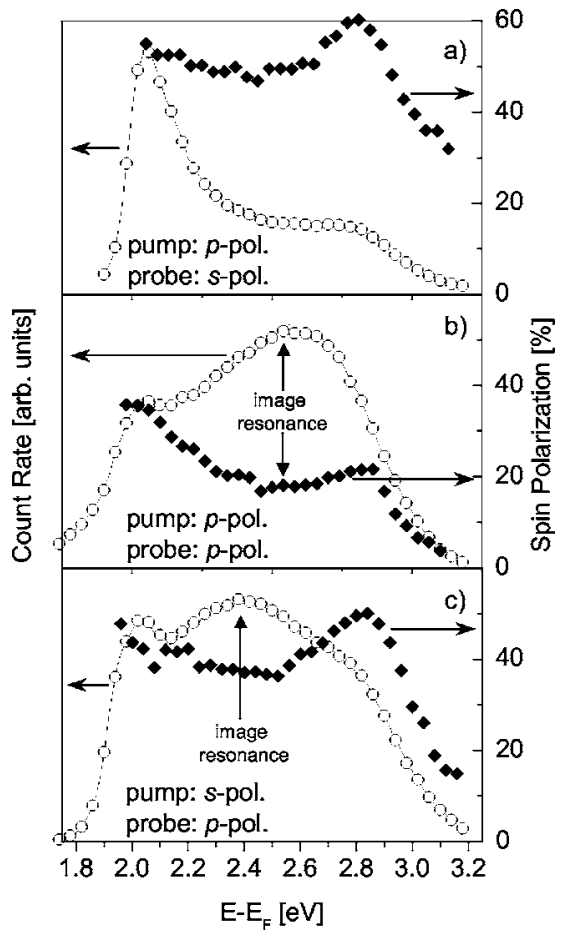

FIG. 7. Bichromatic 2PPE spectra (open circles) and corresponding spin polarization (closed diamonds) of a Cs-covered Co(001) surface obtained with differently polarized UV (pump) and IR (probe) laser pulses at a time delay of $0 \mathrm{fs}$.

to about $35 \%$ in $1 \mathrm{PPE}$ and $45 \%$ in $2 \mathrm{PPE}$ experiments (see Fig. 1). ${ }^{17}$ Furthermore, in the relevant excitation region, the 2PPE initial state of the spin-down (minority) $\Delta_{5}$ band is located rather close to the $\Gamma$ point. Taking into account our above discussion, the excitation from this spin down band is quite unfavorable when compared it to the excitation from the spin up (majority) $\Delta_{5}$ band. Consequently, in contrast to $p$-polarized light, $s$-polarized light should yield a rather high spin polarization in the population of the image resonance. We also have to consider the selection rules regarding the second photoemission step out of the image resonance into the final state. As mentioned above, a probing of the image resonance and the corresponding spin polarization is only possible by $p$-polarized light.

For the present study a pump-probe scheme that enables such measurements has been realized by the use of a bichromatic 2PPE measurement setup in which the frequencydoubled-laser pulse (UV) populates the image resonance, and the fundamental laser pulse (IR) probes this population by excitation above the vacuum level. The light polarization of both pulses, pump and probe, can independently be tuned between $p$ - and $s$-polarization. In Fig. 7, UV-IR 2PPE spectra and the corresponding spin polarizations for different light polarization settings of pump and probe pulses are presented.

The spectrum shown in Fig. 7(a) has been recorded at $s$-polarization of the IR (probe) pulse. Only coupling to bulk states in the final excitation process is possible therefore no image resonance contribution appears in the 2PPE spectrum. Again, the measured spin polarization is governed only by the bulk band structure exhibiting a clear increment around the $\Delta_{5}$ bulk state, as shown before (see Fig. 1).
For the spectra in Fig. 7(b) both UV (pump) and IR (probe) pulses are set to $p$-polarization. Consequently (and in coincidence with Fig. 6), a strong image resonance contribution is observed. Similar to the monochromatic $p$-polarized UV-UV 2PPE results (see Fig. 6), also the spin polarization at the image resonance energy is quenched.

Finally, in Fig. 7(c), the UV (pump) pulse is set to $s$-polarization, and the IR (probe) pulse is set to $p$-polarization. Similar to Fig. 7(b), a large 2PPE yield from the image resonance is present in the spectrum, this time, however, its population has been induced by $s$-polarized light. Therefore, and in contrast to Fig. 7(b), the spin polarization at the surface resonance energy does not decrease at all, but instead behaves very similar to the bulk spin polarization in Fig. 7(a). This observation, together with the data in Fig. 7(b) support the view that the bulk - image resonance transitions govern the spin polarization of the image resonance population. The polarization of the exciting light can obviously be used to select specific transitions and consequently manipulate the degree of spin polarization of the image resonance. We would like to add that in these experiments we have never obtained a spin polarization from the image resonance state higher than the bulk Co polarization values (Fig. 7(a)), as expected. ${ }^{4,27,28}$ To clarify this issue, let us compare the image resonance with the corresponding image state of the noncesiated surface. A distinct image potential state located in front of the surface is "quasi" decoupled from the metal state. This state attenuates fast in the bulk band gap of the metal with the similar periodicity of the wave function as the bulk states at the $X$ point. The Cs induced downward shift induces a stronger coupling of the image state to the bulk bands and consequently a longer decay of its wave function into the bulk, as it approaches the border of the band gap. Finally, in our case the work function is strongly decreased so that the wave function of the image state can effectively overlap with the bulk bands. The periodicity of such a surface resonance wave function can differ from the original image state wave function. As a result, the probability of the optical excitation from the spin down $\Delta_{5}$ band, which is supposed to be small for the image state, probably cannot be neglected any more in the case of the surface resonance.

\section{SUMMARY}

In summary, we have shown that under consideration of corresponding band structure calculations the measured spin polarization and spectral distribution of the $2 \mathrm{PPE}$ from a $\mathrm{Co}(001)$ surface enable an efficient identification of the relevant electronic states and optical selection rules governing the photoemission process.

Correct interpretation may, however, require careful adjustment of the calculated electron DOS to the experimental border conditions such as a finite angle resolution as it is shown in the photoemission from spin up $\Delta_{5}$ bulk states.

Furthermore, we find that the optical selection rules in combination with the adjustable polarization state of the laser light used for our experiments can be utilized to control the spin polarization of the intermediate electron state ad- 
dressed in 2PPE. By this means, we are able to switch the degree of spin polarization of the excited state population of the $\mathrm{Co}(001)$ image state from almost unpolarized to $>40 \%$. Particularly the control of the spin polarization of such surface (or interface) located states may be utilized to optimize the spin injection efficiencies in ferromagnet-semiconductor spintronics devices.

\section{ACKNOWLEDGMENTS}

We thank M. Weinelt and Th. Fauster for insightful conversations. This work was supported in part by the DFG priority program SPP 1133, the European research training network HPRN-CT-2002-00318 ULTRASWITCH, and the DFG research training group 792.
*Electronic address: mkbauer@physik.uni-kl.de

${ }^{1}$ S. A. Wolf, D. D. Awschalom, R. A. Buhrman, J. M. Daughton, S. von Molnár, M. L. Roukes, A. Y. Chtchelkanova, and D. M. Treger, Science 294, 1488 (2001).

${ }^{2}$ I. Zutic, J. Fabian, and S. Das Sarma, Rev. Mod. Phys. 76, 323 (2004)

${ }^{3}$ F. Passek and M. Donath, Phys. Rev. Lett. 69, 1101 (1992).

${ }^{4}$ W. Wallauer and Th. Fauster, Phys. Rev. B 54, 5086 (1996).

${ }^{5}$ M. Lisowski, P. A. Loukakos, A. Melnikov, I. Radu, L. Ungureanu, M. Wolf, and U. Bovensiepen, Phys. Rev. Lett. 95, 137402 (2005).

${ }^{6}$ A. B. Schmidt, M. Pickel, M. Wiemhofer, M. Donath, and M. Weinelt, Phys. Rev. Lett. 95, 107402 (2005).

${ }^{7}$ R. Knorren, K. H. Bennemann, R. Burgermeister, and M. Aeschlimann, Phys. Rev. B 61, 9427 (2000).

${ }^{8}$ P. M. Echenique, R. Berndt, E. V. Chulkov, Th. Fauster, A. Goldmann, and U. Höfer, Surf. Sci. Rep. 52, 219 (2004).

${ }^{9}$ M. Aeschlimann, M. Bauer, S. Pawlik, R. Knorren, G. Bouzerar, and K. H. Bennemann, Appl. Phys. A 71, 1 (2000).

${ }^{10}$ V. P. Zhukov, O. Andreyev, D. Hoffmann, M. Bauer, M. Aeschlimann, E. V. Chulkov, and P. M. Echenique, Phys. Rev. B 70, 233106(R) (2004).

${ }^{11}$ W. Weber, A. Bischof, R. Allenspach, C. H. Back, J. Fassbender, U. May, B. Schirmer, R. M. Jungblut, G. Güntherodt, and B. Hillebrands, Phys. Rev. B 54, 4075 (1996).

${ }^{12}$ A. K. Schmid, D. Atlan, H. Itoh, B. Heinrich, T. Ichinokawa, and J. Kirschner, Phys. Rev. B 48, 2855 (1993), and references therein.
${ }^{13}$ V. Moruzzi, J. Janak, and A. Willams, Calculated Electronic Properties of Metals (Pergamon, New York, 1978).

${ }^{14}$ E. Wimmer, H. Krakauer, M. Weinert, and A. J. Freeman, Phys. Rev. B 24, 864 (1981).

${ }^{15}$ M. Weinert, E. Wimmer, and A. J. Freeman, Phys. Rev. B 26, 4571 (1982).

${ }^{16}$ URL: http://www.flapw.de

${ }^{17}$ M. Aeschlimann, M. Bauer, S. Pawlik, W. Weber, R. Burgermeister, D. Oberli, and H. C. Siegmann, Phys. Rev. Lett. 79, 5158 (1997).

${ }^{18}$ W. Clemens, E. Vescovo, T. Kachel, C. Carbone, and W. Eberhardt, Phys. Rev. B 46, 4198 (1992).

${ }^{19}$ W. Clemens, T. Kachel, O. Rader, E. Vescovo, S. Blügel, C. Carbone, and W. Eberhardt, Solid State Commun. 81, 739 (1992).

${ }^{20}$ Data not shown for brevity, can be obtained upon request.

${ }^{21}$ N. Fischer, S. Schuppler, Th. Fauster, and W. Steinmann, Surf. Sci. 314, 89 (1994).

${ }^{22}$ S. Hüfner, Photoelectron Spectroscopy (Springer-Verlag, Berlin, (2003).

${ }^{23}$ Th. Fauster, Prog. Surf. Sci. 46, 177 (1994).

${ }^{24}$ D. A. Arena, F. G. Curti, and R. A. Bartynski, Phys. Rev. B 56, 15404 (1997).

${ }^{25}$ J. Hermanson, Solid State Commun. 22, 9 (1977).

${ }^{26}$ W. Eberhardt and F. J. Himpsel, Phys. Rev. B 21, 5572 (1980).

${ }^{27}$ U. Thomann, I. L. Shumay, M. Weinelt, and Th. Fauster, Appl. Phys. B 68, 531 (1999).

${ }^{28}$ M. Pickel, A. B. Schmidt, M. Donath, and M. Weinelt, Surf. Sci. (to be published). 